\title{
The Size of Analysis: A Source of Error Affecting Reliability during Maximal Isometric Shoulder Rotation Strength Test-Retest
}

\author{
Chamorro Claudio ${ }^{1,2}$, De la Fuente Carlos ${ }^{1,3 *}$ and Jerez Daniel ${ }^{3}$ \\ ${ }^{1}$ Carrera de Kinesiología, UDA, Cs de la Salud, Facultad de Medicina, Pontificia Universidad Católica de Chile, 7820436 Santiago, Chile \\ ${ }^{2}$ Servicio Kinesiología, Clínica UC San Carlos de Apoquindo, Santiago, Chile \\ ${ }^{3}$ Facultad Cs de la Rehabilitación, Universidad Andrés Bello, Santiago, Chile
}

Submission: March 03, 2018; Published: March 13, 2018

*Corresponding author: Claudio Chamorro, PhD, Pontificia Universidad Católica de Chile, UDA Cs de la salud, Carrera de Kinesiología, Vicuña Mackenna, Chile, Telephone no: +56-9-94398014; Email: cchamorrol@uc.cl

\begin{abstract}
Background: The manner of acquiring strength-testing data may influence peak torque detection and reliability of results in test- retest studies.

Objective: To examine the effects of differing window thresholds on shoulder internal (IR) and external rotators (ER) isometric peak torque standard error of measurements (SEM) and minimal detectable change (MDC) in a test-retest study

Methods: thirty asymptomatic young adults completed dominant shoulder IRI and ER isometric testing using REV 7000. IR and ER were randomly tested twice in $90^{\circ}$ of shoulder abduction in supine position. Raw torque was processed using Mat lab $2014 \mathrm{~b}$ software that computed peak torque at four window thresholds: no window, $75 \%, 50 \%$, and $25 \%$.

Results: One way ANOVA and Bonferroni post hoc test showed similar results in peak torque assessment considering windowing and not windowing data. SEM obtained varied according to the window size considered. Lower SEM and MDC were registered when 50\% and $25 \%$ central data were considered for ER peak torque. There were no variation considering windows sizing for IR peak torque.

Conclusion: Windowing data has to be considered in test-retest peak torque to improve reliability of results. Based on these results we recommend windowing at least $50 \%$ central data in ER peak torque assessment.
\end{abstract}

Keywords: Muscle Dynamometry; Reliability of Results; Shoulder Rotators; Peak Torque; Windows

Abbreviations: ER: External Rotators; IR: Internal Rotators; MDC: Minimal Detectable Change; SEM: Standard Error of Measurements; SPADI: Shoulder Pain and Disability Index

\section{Introduction}

Muscle strength evaluation of the shoulder internal (IR) and external rotators (ER) is often used by clinicians to assess muscle performance and to guide diagnosis and rehabilitation [1]. Factors influencing peak torque and reliability of results in a test- retest design includes the stabilization system, assessment position, similarity in protocol execution and methodological aspects [2,3]. Another factor that has not been extensively studied, is the fact of windowing or not windowing isokinetic data. Full data may oversize peak torque specially at the beginning of the contraction due to overshoot torque when the subject initiated a forceful movement or at the end of the contraction where a significant spike in torque can be also observed [4]. This torque spike occurs as a result of combining a long lever arm with an abrupt end stop during testing. The magnitude of the force fluctuations is quantified in absolute terms as standard error of measurement (SEM) and minimal detectable change (MDC). The SEM quantifies score reliability within indi $\urcorner$ vidual participants on different occasions. To produce a unit-free indicator of SEM error magnitude, the results can be expressed as SEM (\%). MDC, in turn, provides a value range within which a truly unchanged participant score would be expected to remain over repeat testing, at a 95\% CI $[5,6]$. To produce a unit- free indicator, MDC can also be expressed in percentage MDC (\%).The purpose of this study is to examine the effects of differing window thresholds on shoulder internal 
(IR) and external (ER) isometric peak torque, SEM and MDC in a test-retest study.

\section{Material and Methods}

\section{Participants}

A total of 30 asymptomatic participants (22 females and 8 males; aged $23.3 \pm 2.6$ years; body mass index $23.2 \pm 2.6 \mathrm{~kg}$ / $\mathrm{m} 2$; SPADI score $4.1 \pm 5.7$ ) all with right hand dominance were recruited using advertisement in a local university. The inclusion criteria were as follows:

i) Shoulder pain and disability index (SPADI) $\leq 18[7]$

ii) Signed informed consent.

\section{Exclusion criteria included the following}

i) shoulder or neck injury in the last six month

ii) shoulder or neck pain at the moment of assessment history of shoulder or neck surgery orthopedic alterations of the trunk, shoulder, neck or upper limbs

Any condition that would alter the assessment parameters

All of the participants gave written informed consent prior to participation and were able to withdraw from the study at any time without any consequences. The study was approved in accordance with principles established by the Helsinki Declaration by the Scientific Ethical Committee at the School of Medicine of Pontificia Universidad Catolica De Chile Project No. 16-057, date of approval april 212016.

\section{Design}

This test-retest reliability study was performed at the Human Movement Analysis Laboratory of Pontificia Universidad Catolica de Chile between June and July 2017. Peak torque was assessed in asymptomatic university students during two sessions, with a 14 day rest-period between sessions. IR and ER isometric peak torques were tested in a supine position with $90^{\circ}$ shoulder abduction using isokinetic dynamometer REV 7000 (Techno Gym SpA, Gambetola, Forli, Italy). Test order (i.e. IR and ER) was randomly assigned by a computer program assigned by the Statistician software. A trained rater in this device was selected.

\section{Assessment}

The dominant upper limb of participants was assessed. Each participant adopted a supine position. ID was brought to shoulder height and the shoulder-rotation attachment was installed. The arm rested in the rotation cuff pad, with the olecranon approximating the axis of the dynamometer and the participant's hand gripping the input shaft [8]. The machine axis was aligned with the longitudinal axis of the humerus and coincided with the center of the glenohumeral joint. The upper limb was postured as follows: frontal plane at $90^{\circ}$ of abduction; elbow flexed at $90^{\circ}$ in sagittal plane; and forearm in pronation. The arm, thorax, and pelvis were fixed with Velcro straps. The possible anterior translation of the humeral head was also controlled by independent strap. At wrist level the dynamometer was attached through a pulley system placed at $90^{\circ}$ between the dynamometer and participant recording the force, displacement and time signals at $20 \mathrm{~Hz}$. Participants were familiarized with the test by performing two submaximal isometric shoulder rotator contractions lasting 6 seconds (s). Then, three maximal isometric shoulder rotator contractions lasting $6 \mathrm{~s}$ were performed for the IR and ER at $90^{\circ}$ (Figure 1). The rest time between strength tests was $1 \mathrm{~min}$, and the time between contractions was $15 \mathrm{~s}$.

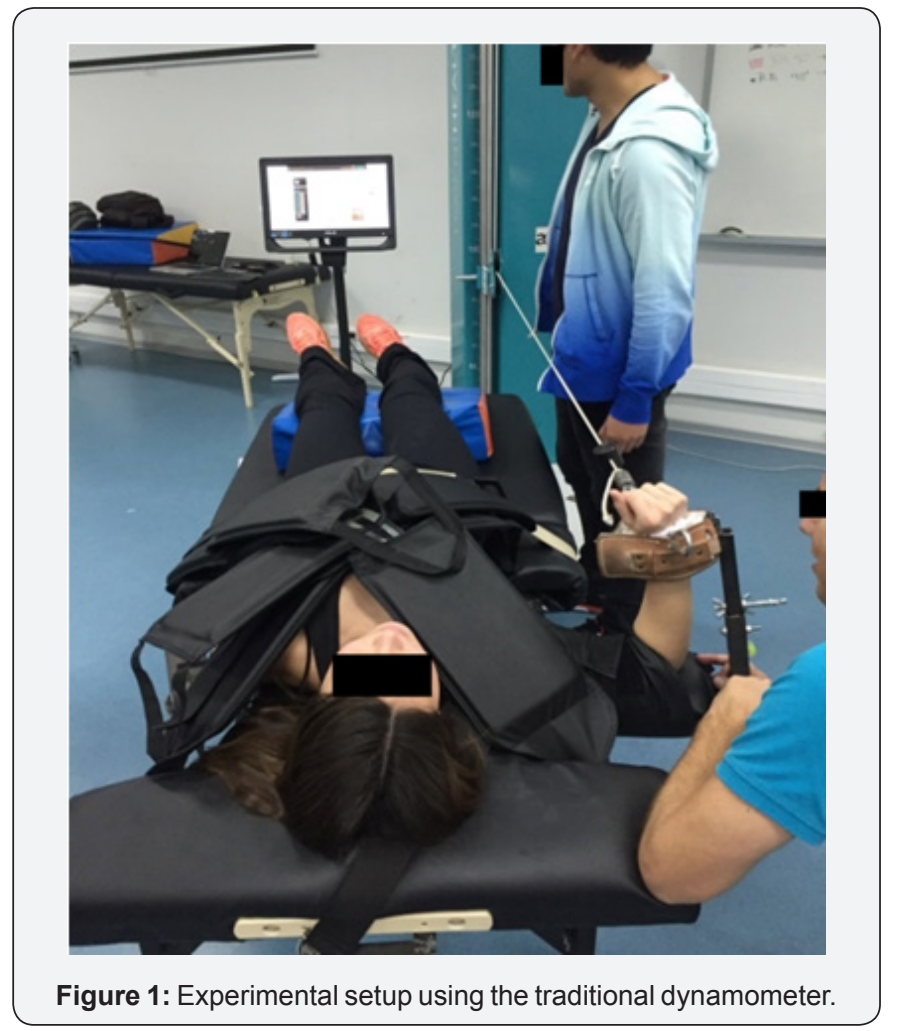

\section{Windows of Analysis}

Raw torque was exported from the dynamometer software and processed using Matlab 2014b software (MathWorks, Inc., Natick, MA, USA) program that computed peak torque at four window thresholds: no window (NW), 75\%, 50\%, and $25 \%$.

\section{Outcomes}

Peak torque, SEM and MDC was determined considering each of the four windows selected.

\section{Data Analysis}

IR and ER peak torque at $90^{\circ}$ of shoulder abduction was used for analysis. Data are expressed as the mean \pm standard deviation. Normal data distribution was confirmed using the Shapiro-Wilk's test $(\mathrm{p}<0.06)$. Homogeneity of variance was confirmed using the Bartlett test $(\mathrm{p}<0.06)$. Analyses of variance with Bonferroni post hoc tests were conducted to compare peak torque determined by each window. Absolute reliability was evaluated to determine intra-participant variations during repeated measurements considering $100,75,50$ and $25 \%$ of central data. Absolute 
reliability was established from SEM obtained as SEM SD $/ \sqrt{2}$, where SD is the standard deviation of differences between sessions. To produce a unit-free indicator of SEM error magnitude, the results of this analysis were expressed as a percentage SEM (\%). Finally, a sensitivity analysis was performed to determine smallest amount of change needed to be considered statistically significant. For this, MDC was calculated at a 95\%CI according to MDC 1.96*SD. The MDC error magnitude was expressed as a unitfree percentage MDC (\%). The unit of measurement error is $\mathrm{Nm}$ [9]. All statistical analyses employed a probability of type I error equal to 0.05 and were performed with the STATA 13 statistical software (SPSS Inc., USA).

Results

Peak torque values comparing windowed to unwindowed isometric data of shoulder IR and ER are presented in Table 1. One way ANOVA and Bonferroni post hoc test showed similar results in peak torque assessment considering windowing and not windowing data. Test- retest absolute reliability results determined by SEM, SEM(\%). MDC and MDC(\%) comparing windowed to unwindowed isokinetic data are presented in Table 2.

Table 1: Test-retest peak torque assessment of IR and ER considering different windowing data.

\begin{tabular}{|c|c|c|c|c|}
\hline Strength tests & NW & 75\% window & 50\% window \\
\hline \multicolumn{3}{|c|}{ Measurement Sessions IR } \\
\hline Session 1, mean \pm sd, Nm & $36.6 \pm 15.5$ & $37.1 \pm 15.6$ & $36.3 \pm 15.2$ \\
\hline Session 2, mean \pm sd, Nm & $36.4 \pm 14.8$ & $36.1 \pm 14.4$ & $35.4 \pm 14.0$ \\
\hline \multicolumn{3}{|c|}{ Measurement Sessions ER } & $36.9 \pm 15.1$ \\
\hline Session 1, mean \pm sd, Nm & $33.1 \pm 13.2$ & $32.8 . \pm 13.1$ & $32.2 \pm 12.9$ \\
\hline Session 2, mean \pm sd, Nm & $33.5 \pm 13.2$ & $33.4 \pm 13.1$ & $32.6 \pm 12.7$ \\
\hline
\end{tabular}

Non-statistical differences were found $(p=0.9)$; Abbreviations: $s d=$ standard deviation.

Table 2: Test-retest absolute reliability results of IR and ER peak torque assessment considering different windowing data.

\begin{tabular}{|c|c|c|c|c|}
\hline Absolute Reliability IR & NW & $\mathbf{7 5 \%}$ window & $\mathbf{5 0 \%}$ window & $\mathbf{2 5 \%}$ window \\
\hline SEM, (Nm) & 3.4 & 3.3 & 3.3 & 3.0 \\
\hline SEM\%, (\%) & 9.2 & 9.1 & 9.1 & 8.4 \\
\hline MDC & 9.41 & 9.2 & 9.2 & 23.4 \\
\hline MDC\% & 24.2 & 25.2 & 25.2 & 1.5 \\
\hline SEM, (Nm) & 2.1 & Absolute Reliability ER & 5.6 & 4.8 \\
SEM\%, (\%) & 6.5 & 2.0 & 5.1 & 1.3 \\
MDC & 6.0 & 6.2 & 15.7 & 13.5 \\
\hline
\end{tabular}

Abbreviations: SEM = standard error of mean; SEM $\%=$ percentage of standard error; $\mathrm{MDC}=$ minimum detectable change; $\mathrm{MDC} \%=$ percentage of minimum detectable change.

\section{Discussion}

No significant difference exists in shoulder IR and ER isometric peak torque testing between windowed and unwindowed data. Previous studies $[10,11]$ have reported that significant difference exists between windowed and non windowed data during peak toque isokinetic testing. Wilk [10] publishes that significant difference exists during isokinetic testing of the shoulder's abductors/adductors at $180^{\circ} / \mathrm{seg}$ and $300^{\circ}$ /segbetween $50 \%$ central windowed data and nonwindowed data. Merritt [12] concludes that significant differences in internal and external rotation peak torque exists considering windowing and unwindowing data at seven different window thresholds. He suggests that with $5 \%$ increments, windowing had very potent effects on peak torque data. Variation in peak torque assessment during isokinetic testing, could be partially explained because movement occurs at a constant predetermined speed in the range of motion referred to as load range where the dynamometer imposes an external load to the limb [13] movement. However, in isokinetic contractions, an exercising limb must free accelerate to the preset speed and decelerate at the end of the exercising range [10]. These phases are performed without the benefit of externally imposed resistance and, consequently, should not be considered during test interpretation [13]. The velocity overshoot is a movement artifact that occurs when the limb accelerates past the desired speed and the dynamometer tries to slow the limb. This generates two effects: 
a) a torque spike (or torque overshoot) as a consequence of the braking mechanism of the dynamometer [11],

b) a short period of time where the angular velocity oscillates until its stabilization at the preset speed [10].

The impact artifact has an effect similar to VO and it is observed during deceleration when the dynamometer begins to slow the lever arm in preparation for stopping at the turnaround point. This causes a torque spike at the end of the repetition due to the lever arm impacting the mechanical end stop [13]. Both VO and impact artifact produce undesirable behavior and are sometimes removed prior to isokinetic test interpretation. By windowing the data, all measure swhich are notobtained at the preset isokinetic angular velocityor at 95 percent of that speed will not be recorded. Isometric contraction is not influenced by angular velocitiy, so peak torque should not be significantly influenced by windowing data as occured in this study. The MDC is a stringent decision limit for establishing improvement/deterioration in peak muscle force or torque following rehabilitation post-injury or as part of a strengthening program in a healthy individual. High heterogeneity between subjects exists for many measure $\neg$ ments in sports medicine, as in the case of peak muscle force. Therefore, SEM and consequently MDC are high [5]. Experts in sports medicine rehabilitation, consider $10 \%$ to $15 \%$ be a clinically relevant improvement or deteriora $\neg$ tion in muscle force. In practice, one criterion for a return to sports is peak muscle strength deficits under $10 \%$ of the contralateral extremity [14]. This small but clinically relevant difference was only detected when 50\% and $25 \%$ of central data ER peak torque were analyzed. ER are susceptible to major fatigue, which could be a source of variability during strength generation tests [15]. To avoid noise to the measurements that can alter the subject performance, the arm, thorax, and pelvis were fixed with Velcro straps. To further control the anterior translation of the humeral head, another Velcro strap was added, but still this could be insufficient to control variability in a better way. Windowing $50 \%$ and $25 \%$ central data helped to reduce in 5\% variability expressed as MDC (\%) in ER peak torque.

Similar MDC (\%) values were found when analyzing different window thresholds on shoulder IR peak torque (25\%). Unlike what occurred with ER, windowing data did not reduce variability. Translated to a rehabilitation program for IR shoulder strengthening, this means that to assure that a strengthening program really improved the subject conditions, at least a $25 \%$ improvement from the initial value should exist to be considered outside the instrument random error, IR strength tests have reported MDC\% ranging from $20-30 \%$, such as in Meeteren et al. [16] when using a Biodex isokinetic dynamometer, as well as Forthomme et al. [17] and Edouard et al. [18] when using Cybex norm isokinetic dynamometer in the scapular plane without windowing data. Clinical relevance of this study is that even though shoulder ER isometric peak torque is not significantly influenced by windowing data, these small changes in peak torque produces relevant improvements in MDC (\%) that needs to be considered when assessing peak torque in test- retest designs.
The main limitations were the use of a convenient sample, an unbalanced gender representation among participants, and the young age of the participants. In conclusion, individual. Small but clinically relevant changes under $20 \%$ are included in the random error of this dynamometer

\section{Conclusion}

Windowing data has to be considered in test-retest peak torque because it is a factor like stabilization system, assessment position, similarity in protocol execution and methodological aspects that can improve reliability of results. Based on these results we recommend windowing at least $50 \%$ central data in ER peak torque assessment to increase test- retest reliability.

\section{Acknowledgement}

The authors would like to thank Melissa A. Sutherland (Fulbright Scholar) for her assistance with manuscript edits and publishing suggestions.

\section{References}

1. Dauty M, Delbrouck C, Huguet D, Rousseau B, Potiron Josse M, et al (2003) Reproducibility of concentric and eccentric isokinetic strength of the shoulder rotators in normal subjects 40 to 55 years old. Isokinet Exerc Sci 11(2): 95-100.

2. de Araujo Ribeiro Alvares JB, Rodrigues R, de Azevedo Franke R, da Silva BG, Pinto RS, et al. (2015) Inter-machine reliability of the Biodex and Cybex isokinetic dynamometers for knee flexor/ extensor isometric, concentric and eccentrictests. Phys Ther Sport 16(1): 59-65.

3. Kim WK, Kim DK, Seo KM, Kang SH (2014) Reliability and validity of isometricknee extensor strength test with hand-held dynamometer depending on its fixation: a pilot study. Ann Rehabil Med 38(1): 84-93.

4. Lavender AP, Nosaka K (2006) Comparison between old and young men for changes in markerts of muscle damage following voluntary eccentric exercise of the elbow Xexors. Appl Physiol Nutr Metabol 31(3): 218-225.

5. Hopkins WG (2000) Measures of reliability in sports medicine and science. Sports Medicine 30(1): 1-15.

6. Atkinson G, Nevill AM (1998) Statistical methods for assessing measurement error (reliability) in variables relevant to sports medicine 26(4): 217-238.

7. Breckenridge JD, Mc Auley JH (2011) Shoulder pain and disability index (SPADI). J Physiother 57(3): 197-200.

8. Beaton DE, Katz JN, Fossel AH, Wright JG, Tarasuk V, et al. (2001) Measuring the wole or the parts?: Validity, reliability, and responsiveness of the disabilities of the arm, shoulder and hand outcome measure in different regions of the upper extremity. J Hand Ther 14(2): 128-146.

9. Johansson FR, Cools AM, Skillgate E, Lapauw ML, Deneulin VP, et al (2014) Measuring eccentric strength of the shoulder external rotators using a hand-held dynamometer: reliability and validity. Br J Sports Med 50(7): 719-725.

10. Sapega AA, Nicholas A, Sokolow D, Saraniti A (1982) The nature of torque "overshoot" in Cybex isokinetic dynamometry. Med Sci Sports Exerc 14(5): 368-375.

11. Wilk KE, Arrigo CA, Andrews JR (1992) Isokinetic testing of the shoulder abductorsand adductors: windowed vs nonwindowed data collection. J Orthop Sports Phys Ther 15(2): 107-112. 
12. Merrit AC, Rienann BL George JD (2016) To Window or Not To Window? The Effects of Window Thresholds On Shoulder Isokinetic Testing. Med Sci Sports Exerc 48(5S): 718-723.

13. Peruzzo FS, Bottaro M, Celes RS, Brown LE, Assis de Oliveira F (2010) The influence of velocity overshoot movement artifact on isokinetic knee extension tests. J Sports Sci Med 9: 140-146.

14. Prentice, William E (2004) Rehabilitation techniques for sports medicine and athletic training, McGraw-hill, New York, USA.

15. Bassett RW, Browne AO, Morrey BF, An KN (1990) Glenohumeral muscle force and moment mechanics in a position of shoulder instability. J Biomech 23(5): 405-415.
16. Meeteren JV, Roebroeck ME, Stam HJ (2002) Test-retest reliability in isokinetic muscle strength measurements of the shoulder. J Rehabil Med 34(2): 91-95.

17. Forthomme B, Dvir Z, Crielaard JM, Croisier JL (2011) Isokinetic assessment of the shoulder rotators: a study of optimal test position. Clin Physiol Funct Imaging 31(3): 227-232.

18. Edouard P, Codine P, Samozino P, Bernard PL, Herisson C, et al. (2013) Reliability of shoulder rotators isokinetic strength imbalance measured using the Biodex dynamometer. J Sport Sci Med 16(2): 162165.

\section{Your next submission with Juniper Publishers will reach you the below assets}

- Quality Editorial service

- Swift Peer Review

- Reprints availability

- E-prints Service

- Manuscript Podcast for convenient understanding

- Global attainment for your research

- Manuscript accessibility in different formats

( Pdf, E-pub, Full Text, Audio)

- Unceasing customer service

Track the below URL for one-step submission https://juniperpublishers.com/online-submission.php 